\title{
Focusing of electromagnetic waves into a dielectric slab. II. Numerical results
}

Velauthapillai Dhayalan
Faculty of Engineering, University College of Bergen, Nygårdsgt 112, N-5020 Bergen, Norway

Department of Physics and Technology, University of Bergen, Allègt. 55, N-5007 Bergen, Norway

Based on existing exact and asymptotic Kirchhoff solutions for focused electromagnetic fields inside a dielectric slab we present numerical comparisons between them also for the special cases of focusing through a single interface or in a single medium. These comparisons show that the exact and asymptotic Kirchhoff solutions for focusing in a single medium or through a single interface agree well, except at observation points near to the interface, while a small difference between the two solutions for the focused electrical field inside a dielectric slab is observed, especially at observation points near to one of the interfaces. This difference is believed to be due to contributions from surface waves, which are not accounted for in the asymptotic Kirchhoff solutions. At low Fresnel numbers focal shift phenomena are observed in all three cases. [D0I: 10.2971/jeos.2011.11036]

Keywords: Focusing, focal shift, interface, slab, asymptotic and exact results

\section{INTRODUCTION}

Focusing of electromagnetic waves in a layered medium of different refractive indices is of interest in many different applications, such as optical data storage $[1,2]$ and hyperthermia treatment $[3,4]$. The first investigation of such focusing problems was carried out by Ling and Lee [3], who considered focusing of electromagnetic waves from air through a plane interface of a dielectric. To save computing time their computations of focused fields were based on asymptotic solutions with restricted range of validity. But their approach was based on the physical-optics or Kirchhoff approximation, making the results valid also at low Fresnel numbers. Flagello et al. [2] considered focusing in a layered medium, and Török et al. [5-8] considered essentially the same problem as in [3], but based on the Debye approximation, implying that their results are valid only at sufficiently high Fresnel numbers.

In focusing at low Fresnel numbers of scalar [9]-[14] as well as electromagnetic waves [3,15] asymmetries about the geometrical focal plane and associated focal-shift phenomena have been found theoretically in the Kirchhoff approximation, but not in the Debye approximation. Since corresponding asymmetries and focal shifts have been observed experimentally, both for electromagnetic [16] and scalar waves [13, 17], one can conclude that the Debye approximation is invalid at low Fresnel numbers. In spite of this the Debye approximation is often used instead of the Kirchhoff aproximation because the former may provide a significant reduction in computing time [18]. But in practical designs of low-Fresnel number focusing systems where it is important to know the precise position of maximum energy concentration, the Kirchhoff approximation is inevitable. Such precise knowledge can be of critical importance when electromagnetic waves are focused inside a thinly layered medium. Optimization of focused fields and shaping of the focused intensity distribution are of interest in fields such as photolithography, microscopy, and materials science [19-25].

To enable one to study the effects of focusing in a thin layer, exact Kirchhoff solutions for focused electromagnetic fields inside a dielectric slab were presented in [26], where also corresponding exact solutions for reflected and transmitted fields were given. Asymptotic Kirchhoff solutions for the focused field inside the slab were obtained by expressing the impulse response of the problem as a plane-wave expansion and evaluating the corresponding exact $\left(k_{x}, k_{y}\right)$ integral by the method of stationary phase [26].

For focusing of two-dimensional (2D) electromagnetic waves [27]-[29] Kirchhoff solutions were shown to be very accurate, both for low Fresnel numbers and high angular apertures. Numerical results based on Kirchhoff solutions were compared to experimental results for focusing of $2 \mathrm{D}$ waves through a plane dielectric interface and good agreement was found [30, 31].

The paper is organized as follows. For the sake of completeness, we summarize in section 2 exact and asymptotic Kirchhoff solutions for focused electromagnetic fields inside a slab due to a time-harmonic linearly polarized aperture current. Numerical techniques for computing focused fields inside the slab are discussed in section 3 . In section 4 , exact and asymptotic Kirchhoff results are presented for electromagnetic fields obtained by focusing in a single medium, through a single interface, and into a dielectric slab. Our findings are summarized in section 5 . 


\section{Focusing into a slab}

\subsection{Exact results}

Consider an electromagnetic wave $\left(\mathbf{E}^{i}, \mathbf{H}^{i}\right)$ that is incident upon a slab with interfaces at $z=z_{0}$ and $z=z_{1}$. The permittivity, permeability, and conductivity are $\epsilon_{0}, \mu_{0}$, and $\sigma_{0}$ in the half-space $z \leq z_{0}, \epsilon_{1}, \mu_{1}$, and $\sigma_{1}$ inside the slab $\left(z_{0} \leq z \leq z_{1}\right)$, and $\epsilon_{2}, \mu_{2}$, and $\sigma_{2}$ in the half-space $z \geq z_{1}$. In [26] analytical results were presented for the electromagnetic field transmitted into the slab in terms of a given field or source current in the region $z<z_{0}$ producing the incident field $\left(\mathbf{E}^{i}, \mathbf{H}^{i}\right)$. In this paper the incident wave is assumed to be due to a timeharmonic, $x$ polarized source current in the aperture plane $z=0$ that produces a converging wave with focus at the point $\left(0, z_{f}\right)$ inside the slab. In accordance with the physical-optics or Kirchhoff approximation the source current is assumed to vanish outside the aperture area $\mathcal{A}$ in Fig. 1 and is assumed to have an amplitude distribution $A\left(x^{\prime}, y^{\prime}\right)$ and a phase distribution $\phi\left(x^{\prime}, y^{\prime}\right)$ inside $\mathcal{A}$. With these assumptions the exact Kirchhoff solution for the focused electric field inside the slab is given by [26]

$$
\begin{gathered}
\mathbf{E}(\mathbf{r}, t)=\Re\left\{\mathbf{E}(\mathbf{r}) e^{-i \omega t}\right\} ; \mathbf{E}(\mathbf{r})=\mathbf{E}(\mathbf{r})^{+}+\mathbf{E}(\mathbf{r})^{-} \\
\mathbf{E}^{ \pm}(\mathbf{r})=\mathbf{E}^{T E \pm}(\mathbf{r})+\mathbf{E}^{T M \pm}(\mathbf{r}), \\
\mathbf{E}^{p \pm}(\mathbf{r})=\iint_{\mathcal{A}} A\left(x^{\prime}, y^{\prime}\right) \exp \left[-i \phi\left(x^{\prime}, y^{\prime}\right)\right] \\
\times \mathbf{F}^{p \pm}\left(x^{\prime}, y^{\prime}\right) d x^{\prime} d y^{\prime} \quad(p=T E, T M),
\end{gathered}
$$

where the impulse response $\mathbf{F}^{p \pm}\left(x^{\prime}, y^{\prime}\right)$ is given by the spectral integral

$$
\mathbf{F}^{p \pm}\left(x^{\prime}, y^{\prime}\right)=\iint_{-\infty}^{\infty} \mathbf{f}^{p \pm}\left(k_{x}, k_{y}\right) \exp \left[i h^{ \pm}\left(k_{x}, k_{y}\right)\right] d k_{x} d k_{y}
$$

with

$$
\begin{gathered}
h^{ \pm}\left(k_{x}, k_{y}\right)=k_{x}\left(x-x^{\prime}\right)+k_{y}\left(y-y^{\prime}\right)+k_{z_{0}} z_{0} \pm k_{z_{1}}\left(z-z_{0}\right), \\
\mathbf{f}^{T M \pm}=\frac{\omega \mu_{0}}{\pi c^{2} k_{0} k_{1}} T^{T M \pm} \frac{k_{x}}{k_{t}^{2}} \mathbf{k}^{ \pm} \times\left(\mathbf{k}_{t} \times \hat{\mathbf{e}}_{z}\right), \\
\mathbf{f}^{T E \pm}=\frac{\omega \mu_{0}}{\pi c^{2}} T^{T E \pm} \frac{k_{y}}{k_{z_{0}} k_{t}^{2}} \mathbf{k}_{t} \times \hat{\mathbf{e}_{\mathbf{z}}} \\
\mathbf{k}^{ \pm}=\mathbf{k}_{t} \pm k_{z_{1}} \hat{\mathbf{e}}_{z} ; \mathbf{k}_{t}=k_{x} \hat{\mathbf{e}}_{x}+k_{y} \hat{\mathbf{e}}_{y}, \\
k_{z_{j}}=\sqrt{k_{j}^{2}-k_{t}^{2}} ; k_{j}^{2}=\left(\frac{\omega}{c}\right)^{2} \mu_{j}\left(\epsilon_{j}+\frac{4 \pi i \sigma_{j}}{\omega}\right) \quad(j=0,1,2),
\end{gathered}
$$

where the permittivity, permeability, and conductivity of medium $j$ are given by $\epsilon_{j}, \mu_{j}$, and $\sigma_{j}$, respectively. $\mathbf{E}^{p+}$ and $\mathbf{E}^{p-}$ are electric fields comprised of plane waves having wave vectors with positive and negative $z$ components, respectively.

The transmission coefficients $T^{p \pm}(p=T E, T M)$ in (5)-(6) are given by

$$
\begin{gathered}
T^{p+}=\frac{t_{01}^{p}}{1+r_{01}^{p} r_{12}^{p} \exp \left(i 2 \delta_{1}\right)} ; T^{p-}=T^{p+} r_{12}^{p} e^{i 2 \delta_{1}} \\
\delta_{1}=k_{z_{1}}\left(z_{1}-z_{0}\right),
\end{gathered}
$$

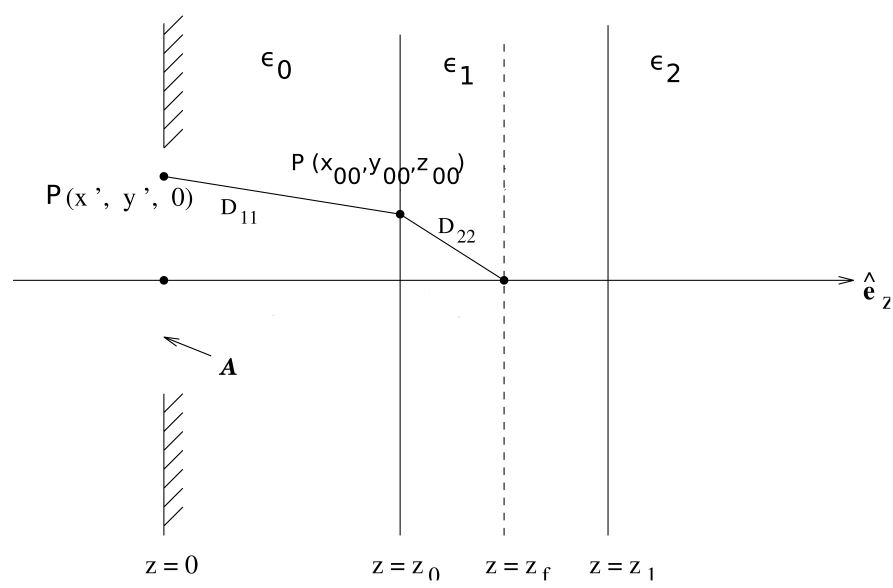

FIG. 1 Focusing into a dielectric slab. The focal plane is at $z=z_{f}$.

where $r_{m n}$ and $t_{m n}(m, n=0,1,2)$ are Fresnel reflection and transmission coefficients for a single interface, given by

$$
\begin{gathered}
r_{m n}^{p}=\frac{q_{m}^{p}-q_{n}^{p}}{q_{m}^{p}+q_{n} p} ; q_{j}^{T M}=\frac{\mu_{j} k_{z_{j}}}{k_{j}^{2}} ; \\
q_{j}^{T E}=\frac{k_{z_{j}}}{\mu_{j}} \quad(j=m, n), \\
t_{m n}^{T M}=\frac{2 k_{m} \mu_{n}}{k_{n} \mu_{m}} \frac{q_{m}^{T M}}{q_{m}^{T M}+q_{n}^{T M}} ; t_{m n}^{T E}=\frac{2 q_{m}^{T E}}{q_{m}^{T E}+q_{n}^{T E}} .
\end{gathered}
$$

We let the amplitude of the aperture current distribution be uniform, i.e. $A\left(x^{\prime}, y^{\prime}\right)=1$, and let the phase $\phi\left(x^{\prime}, y^{\prime}\right)$ be determined from geometrical optics so as to cancel the aberrations introduced on refraction through the first interface of the slab. Figure 1 shows that the requirement of equal phase at the focal point $\left(0, z_{f}\right)$ inside the slab for fields along all geometrical rays from different source points $\left(x^{\prime}, y^{\prime}, 0\right)$ via different refraction points $\left(x_{00}, y_{00}\right)$ at the interface $z=z_{0}$ is given by

$$
\phi\left(x^{\prime}, y^{\prime}\right)=k_{0} D_{11}+k_{1} D_{22}-k_{0} z_{0}-k_{1}\left(z_{f}-z_{0}\right) ，
$$

where $D_{11}$ is the distance from a source point $\left(x^{\prime}, y^{\prime}, 0\right)$ in the aperture plane to the corresponding refraction point $\left(x_{00}, y_{00}, z_{00}\right)$ at the first interface, and $D_{22}$ is the distance from $\left(x_{00}, y_{00}, z_{00}\right)$ to the focal point $\left(0,0, z_{f}\right)$, i.e. (see Fig. 1$)$

$$
\begin{aligned}
& D_{11}=\left[\left(x_{00}-x^{\prime}\right)^{2}+\left(y_{00}-y^{\prime}\right)^{2}+z_{0}^{2}\right]^{1 / 2} \\
& D_{22}=\left[x_{00}^{2}+y_{00}^{2}+\left(z_{f}-z_{0}\right)^{2}\right]^{1 / 2} .
\end{aligned}
$$

All numerical results presented in this paper were obtained using the phase $\phi\left(x^{\prime}, y^{\prime}\right)$ given in (12).

\subsection{Asymptotic results}

As indicated in Fig. 2, the asymptotic solution for the spectral integral $\mathbf{F}^{p+}$ in (3) is obtained by exploiting the fact that the main contribution to each of these impulse-response integrals ( $p=T E, T M$ ) comes from one particular plane wave emitted by the aperture point $\left(x^{\prime}, y^{\prime}, 0\right)$, namely that plane wave for which the corresponding ray on refraction through the first interface of the slab at the point $\left(x_{0}, y_{0}, z_{0}\right)$ passes through the observation point $(x, y, z)[3,26,32]$. Thus, by evaluating each of the integrals in (3) asymptotically using the method of sta- 


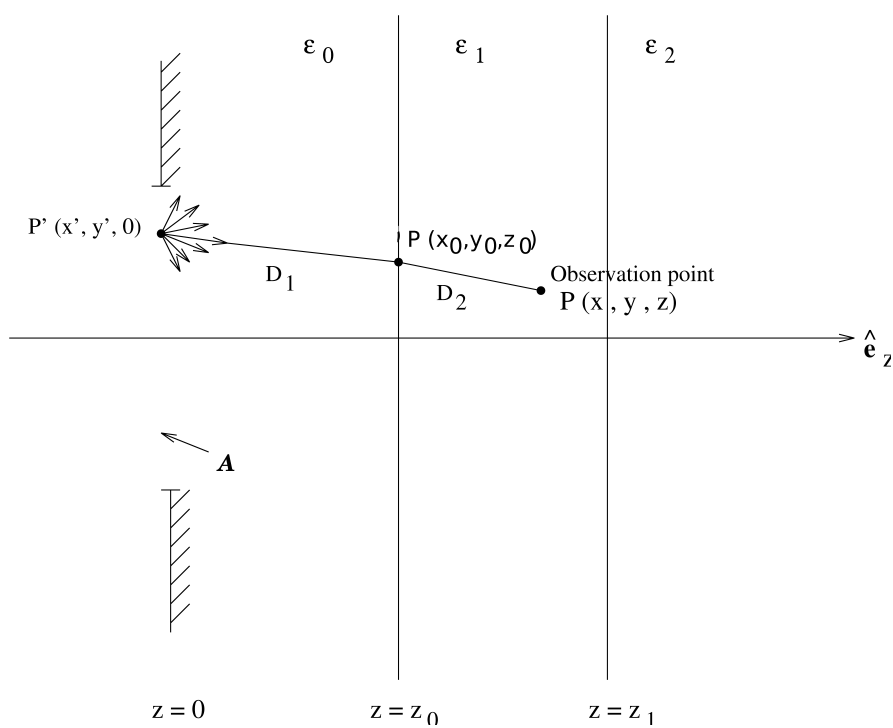

FIG. 2 Refraction through a single dielectric interface. The main contribution from the source point $P^{\prime}\left(x^{\prime}, y^{\prime}, 0\right)$ is due to the plane wave associated with the geometrical ray that passes from the source point $P^{\prime}\left(x^{\prime}, y^{\prime}, 0\right)$ via the refraction point $P\left(x_{0}, y_{0}, z_{0}\right)$ to the observation point $P(x, y, z)$.

tionary phase, we obtain [26]

$$
\begin{aligned}
\mathbf{F}^{p+}\left(x^{\prime}, y^{\prime}\right) \sim & -2 \pi i k_{0} z_{0} \frac{D}{D_{1}^{2}} \mathbf{f}^{p+}\left(k_{x}^{s}, k_{y}^{s}\right) \\
& \times \exp \left[i\left(k_{0} D_{1}+k_{1} D_{2}\right)\right]
\end{aligned}
$$

where $k_{x}^{s}$ and $k_{y}^{s}$ are given by

$$
\begin{aligned}
& k_{x}^{s}=k_{0} \frac{x_{0}-x^{\prime}}{D_{1}}=k_{1} \frac{x-x_{0}}{D_{2}} \\
& k_{y}^{s}=k_{0} \frac{y_{0}-y^{\prime}}{D_{1}}=k_{1} \frac{y-y_{0}}{D_{2}} .
\end{aligned}
$$

In (14), $D_{1}$ is the distance from the source point $\left(x^{\prime}, y^{\prime}, 0\right)$ to the refraction point $\left(x_{0}, y_{0}, z_{0}\right)$ and $D_{2}$ is the distance from $\left(x_{0}, y_{0}, z_{0}\right)$ to the observation point $(x, y, z)$, i.e.

$$
\begin{aligned}
& D_{1}=\left[\left(x_{0}-x^{\prime}\right)^{2}+\left(y_{0}-y^{\prime}\right)^{2}+z_{0}^{2}\right]^{1 / 2} \\
& D_{2}=\left[\left(x-x_{0}\right)^{2}+\left(y-y_{0}\right)^{2}+\left(z-z_{0}\right)^{2}\right]^{1 / 2},
\end{aligned}
$$

and the quantity $D$ is given by

$$
D=\frac{1}{\left[1+D_{2} / \rho_{1}\right]^{1 / 2}} \frac{1}{\left[1+D_{2} / \rho_{2}\right]^{1 / 2}},
$$

with

$$
\rho_{1}=\frac{k_{1} D_{1}}{k_{0}} ; \rho_{2}=\frac{k_{1} D_{1}^{3}\left(z-z_{0}\right)^{2}}{k_{0} z_{0}^{2} D_{2}^{2}} .
$$

Before proceeding with the asymptotic solution for the spectral integral $\mathbf{F}^{p-}$, we add the exponential factor $\exp \left(i 2 \delta_{1}\right)$ of the transmission coefficient $T^{p-}$ in (9) to $h^{-}\left(k_{x}, k_{y}\right)$ in (3) and (4) to obtain

$$
\begin{aligned}
\mathbf{F}^{p-}\left(x^{\prime}, y^{\prime}\right)= & \iint_{-\infty}^{\infty} \mathbf{f}_{M}^{p-}\left(k_{x}, k_{y}\right) \\
& \times \exp \left[i h_{M}^{-}\left(k_{x}, k_{y}\right)\right] d k_{x} d k_{y},
\end{aligned}
$$

where

$$
\mathbf{f}_{M}^{p-}\left(k_{x}, k_{y}\right)=\mathbf{f}^{p-}\left(k_{x}, k_{y}\right) r_{12}^{p} \frac{T^{p+}}{T^{p-}}
$$

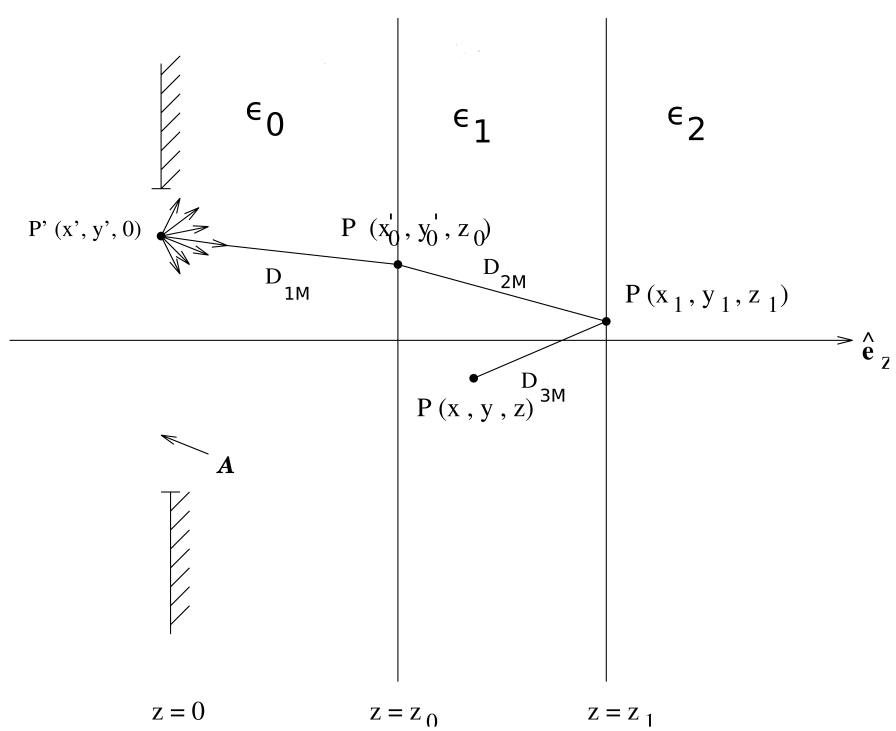

FIG. 3 Refraction through the first interface and reflection from the second interface of the slab. The main contribution to $\mathbf{F}^{p-}\left(x^{\prime}, y^{\prime}\right)$ in (3) from the source point $P^{\prime}\left(x^{\prime}, y^{\prime}, 0\right)$ is due to the plane wave associated with the geometrical ray that passes through the observation point $P(x, y, z)$ after refraction through the first interface of the slab at $P\left(x_{0}, y_{0}, z_{0}\right)$ and reflection from the second interface at $P\left(x_{1}, y_{1}, z_{1}\right)$.

$$
\begin{aligned}
h_{M}^{-}\left(k_{x}, k_{y}\right)= & k_{x}\left(x-x^{\prime}\right)+k_{y}\left(y-y^{\prime}\right)+k_{z_{0}} z_{0} \\
& +k_{z_{1}}\left(d+z_{1}-z\right),
\end{aligned}
$$

with $d=z_{1}-z_{0}$ being the thickness of the slab. The stationary points $\left(k_{x M}^{s}, k_{y M}^{s}\right)$ of the phase function $h_{M}^{-}\left(k_{x}, k_{y}\right)$ are given by [26]

$$
\begin{aligned}
& k_{x M}^{s}=k_{0} \frac{x_{0}^{\prime}-x^{\prime}}{D_{1 M}}=k_{1} \frac{x_{1}-x_{0}^{\prime}}{D_{2 M}}=k_{1} \frac{x-x_{1}}{D_{3 M}}, \\
& k_{y M}^{s}=k_{0} \frac{y_{0}^{\prime}-y^{\prime}}{D_{1 M}}=k_{1} \frac{y_{1}-y_{0}^{\prime}}{D_{2 M}}=k_{1} \frac{y-y_{1}}{D_{3 M}} .
\end{aligned}
$$

Here $D_{1 M}$ is the distance between a source point $\left(x^{\prime}, y^{\prime}, 0\right)$ in the aperture plane and the refraction point $\left(x_{0}^{\prime}, y_{0}^{\prime}, z_{0}\right)$ at the first interface, $D_{2 M}$ is the distance between $\left(x_{0}^{\prime}, y_{0}^{\prime}, z_{0}\right)$ and the reflection point $\left(x_{1}, y_{1}, z_{1}\right)$ at the second interface, and $D_{3 M}$ is the distance from $\left(x_{1}, y_{1}, z_{1}\right)$ to the observation point $(x, y, z)$ inside the slab, as shown in Fig. 3. Thus,

$$
\begin{gathered}
D_{1 M}=\left[\left(x_{0}^{\prime}-x^{\prime}\right)^{2}+\left(y_{0}^{\prime}-y^{\prime}\right)^{2}+z_{0}^{2}\right]^{1 / 2} \\
D_{2 M}=\left[\left(x_{1}-x_{0}^{\prime}\right)^{2}+\left(y_{1}-y_{0}^{\prime}\right)^{2}+d^{2}\right]^{1 / 2}, \\
D_{3 M}=\left[\left(x-x_{1}\right)^{2}+\left(y-y_{1}\right)^{2}+\left(z-z_{1}\right)^{2}\right]^{1 / 2} .
\end{gathered}
$$

Using the lowest-order term in equation (9.7a) in [32], we find the asymptotic contribution to $\mathbf{F}^{p-}\left(x^{\prime}, y^{\prime}\right)$ in (19) to be

$$
\begin{aligned}
\mathbf{F}^{p-}\left(x^{\prime}, y^{\prime}\right) \sim & -2 \pi i k_{0} z_{0} \frac{D_{M}}{D_{1 M}^{2}} \mathbf{f}_{M}^{p-}\left(k_{x M}^{s}, k_{y M}^{s}\right) \\
& \times \exp \left[i\left(k_{0} D_{1 M}+k_{1} D_{2 M}+k_{1} D_{3 M}\right],\right.
\end{aligned}
$$

where $D_{M}$ is given by

$$
D_{M}=\frac{1}{\left(1+\frac{D_{2 M}+D_{3 M}}{\rho_{1 M}}\right)^{1 / 2}} \frac{1}{\left(1+\frac{D_{2 M}+D_{3 M}}{\rho_{2 M}}\right)^{1 / 2}},
$$

with

$$
\rho_{1 M}=\rho_{1}=\frac{k_{1} D_{1}}{k_{0}} ; \rho_{2 M}=\frac{k_{1} D_{1 M}^{3} d^{2}}{k_{0} z_{0}^{2} D_{2 M}^{2}} .
$$


As indicated in Fig. 3, the asymptotic solution for $\mathbf{F}^{p-}\left(x^{\prime}, y^{\prime}\right)$ comes from that particular plane wave emitted by the source point $\left(x^{\prime}, y^{\prime}, 0\right)$ in the aperture which on refraction through the first interface at $\left(x_{0}^{\prime}, y_{0}^{\prime}, z_{0}\right)$ and reflection from the second interface at $\left(x_{1}, y_{1}, z_{1}\right)$ passes through the observation point $(x, y, z)$.

\section{Numerical Techniques}

\subsection{Asymptotic Kirchhoff results}

To provide asymptotic results for $\mathbf{F}^{p+}\left(x^{\prime}, y^{\prime}\right)$ in (14) for a particular observation point $(x, y, z)$, the quantities $D_{1}$ and $D_{2}$ in (16) must be determined. This was accomplished by finding the corresponding refraction point $\left(x_{0}, y_{0}, z_{0}\right)$ at the first interface in Fig. 2 by use of Fermat's priciple. To that end, we implemented a two-dimensional Newton's method for nonlinear systems of equations to find the unknown variables $x_{0}$ and $y_{0}$. We employed numerical algorithms that combined the rapid convergence of Newton's method with a globally convergent strategy, as explained in [33]. The same approach was used to determine the quantities $D_{11}$ and $D_{22}$ in (13) (Fig. 1) and to provide asymptotic results for $\mathbf{F}^{p-}\left(x^{\prime}, y^{\prime}\right)$ in (19), in which case determination of the quantities $D_{1 M}, D_{2 M}$, and $D_{3 M}$ in (24) and (25) (Fig. 3) were required. The integrals in (2) were computed using the SSP method $[32,34]$ for double integrals.

\subsection{Exact Kirchhoff results}

The numerical techniques used to obtain exact results for the focused electric field inside a slab are similar to those employed previously [35] for focusing of electromagnetic waves through a plane interface. First, the spectral double integral in (3) is computed to provide the contribution due to one source point $\left(x^{\prime}, y^{\prime}, 0\right)$ in the aperture. Second, the double integration over the aperture plane in (2) is carried out. This quadruple integration procedure is quite time consuming. Therefore, we reduce the spectral double integral in (3) to a single integral by making the change of integration variables

$$
k_{x}=k_{1} t \cos \beta ; k_{y}=k_{1} t \sin \beta,
$$

writing

$$
x-x^{\prime}=\rho \cos \phi ; y-y^{\prime}=\rho \sin \phi,
$$

and using the formulas in equations (A1)-(A4) in [35] to obtain from (2)-(3) and (9)

$$
\begin{gathered}
\mathbf{E}^{ \pm}=\mathbf{E}^{T E \pm}+\mathbf{E}^{T M \pm}=\iint_{A} A\left(x^{\prime}, y^{\prime}\right) \exp \left[-i \phi\left(x^{\prime}, y^{\prime}\right)\right] \\
\times \mathbf{F}^{ \pm}\left(x^{\prime}, y^{\prime}\right) d x^{\prime} d y^{\prime},
\end{gathered}
$$

where

$$
\begin{aligned}
\mathbf{F}^{ \pm}\left(x^{\prime}, y^{\prime}\right)=k_{1}^{2} \int_{0}^{\infty} & {\left[\mathbf{B}^{ \pm}(t) J_{0}\left(\beta_{1} t\right)+\mathbf{D}^{ \pm}(t) \frac{2 J_{1}\left(\beta_{1} t\right)}{\beta_{1} t}\right] } \\
& \times \frac{\exp \left[i \psi^{ \pm}(t)\right]}{k_{z_{1}}} t d t
\end{aligned}
$$

with

$$
\begin{gathered}
\psi^{+}(t)=k_{z_{0}} z_{0}+k_{z_{1}}\left(z-z_{0}\right) \\
\psi^{-}(t)=k_{z_{0}} z_{0}+k_{z_{1}}\left(d+z_{1}-z\right)
\end{gathered}
$$

$$
k_{z_{0}}=k_{1}\left(k_{r}^{2}-t^{2}\right)^{\frac{1}{2}} ; k_{r}=\frac{k_{0}}{k_{1}} ; k_{z_{1}}=k_{1}\left(1-t^{2}\right)^{\frac{1}{2}}
$$

In (32), $\beta_{1}=k_{1} \rho=k_{1}\left[\left(x-x^{\prime}\right)^{2}+\left(y-y^{\prime}\right)^{2}\right]^{\frac{1}{2}}$ and $J_{0}$ and $J_{1}$ are cylindrical Bessel functions of order zero and one, respectively. The components of $\mathbf{B}^{ \pm}(t)$ and $\mathbf{D}^{ \pm}(t)$ are listed in Appendix A.

\subsubsection{Subdivision of spectral integral}

We assume that the media outside the slab are equal, so that $\epsilon_{2}=\epsilon_{0}, \mu_{2}=\mu_{0}$, and $\sigma_{2}=\sigma_{0}$, and that the media inside and outside the slab have no absorption $\left(\sigma_{0}=\sigma_{1}=0\right)$, implying that both $k_{0}$ and $k_{1}$ are real. Then $k_{z_{0}}$ or $k_{z_{1}}$ has either real or imaginary values in the whole integration interval in (32). Further, we assume that the medium inside the slab is optically denser than the medium surrounding it, so that $k_{1}>k_{0}$. Then, with $k_{r}=k_{0} / k_{1}<1$, both $k_{z_{0}}$ and $k_{z_{1}}$ in (34) are real in the interval $0 \leq t<k_{r} ; k_{z_{0}}$ is imaginary and $k_{z_{1}}$ is real in the interval $k_{r}<t<1$; and both $k_{z_{0}}$ and $k_{z_{1}}$ are imaginary in the interval $1<t<\infty$. The integrand in (32) is well-behaved in each of these three intervals, but not at $t=k_{r}$ or at $t=1$. Hence we divide the integral in (32) in three different parts by writing

$$
\mathbf{F}^{ \pm}=\mathbf{F}_{H 1}^{ \pm}+\mathbf{F}_{H 2}^{ \pm}+\mathbf{F}_{I}^{ \pm},
$$

where $\mathbf{F}_{H 1}^{ \pm}, \mathbf{F}_{H 2}^{ \pm}$, and $\mathbf{F}_{I}^{ \pm}$represent integrations over the intervals $\left[0, k_{r}\right],\left[k_{r}, 1\right]$, and $[1, \infty]$, respectively. Here the integrands of $\mathbf{F}_{H 1}^{ \pm}$and $\mathbf{F}_{H 2}^{ \pm}$are comprised of homogeneous plane waves inside the slab, while the integrands of $\mathbf{F}_{I}^{+}$and $\mathbf{F}_{I}^{-}$are comprised of evanescent plane waves.

\subsubsection{Integration techniques}

Exact results for a given observation point $(x, y, z)$ were obtained by first computing the spectral integrals $\mathbf{F}^{p \pm}\left(x^{\prime}, y^{\prime}\right)$ in (32), and then subsituting the results into the integrands of (2). The whole integration interval in (32) was covered by dividing it into several subintervals, as explained in section 3.1. The integration interval for each of $\mathbf{F}_{H 1}^{ \pm}, \mathbf{F}_{H 2}^{ \pm}$, and $\mathbf{F}_{I}^{ \pm}$ in (35) was further divided in subintervals, and the integrations were carried as discussed in Appendices B and C. The approach to compute each of these integrals was to use the Gauss-Legendre method [36] for cases in which the integrand contained no rapidly oscillating exponential function and to use the SSP (Stamnes-Spelkavik-Pedersen) method [32, 34] for cases in which the integrand was a product of an amplitude function and an oscillating exponential function that varied faster than the amplitude function. Also, asymptotic formulas for the Bessel functions in (32) were used to further reduce the computing time. The integrals in (2) were then computed using the SSP method $[32,34]$ for double integrals. Although we were able to reduce the computing time using these efficient algorithms for numerical evaluation, the computing time for obtaining exact numerical results was still about one thousand times longer than the computing time needed to obtain asymptotic results. 

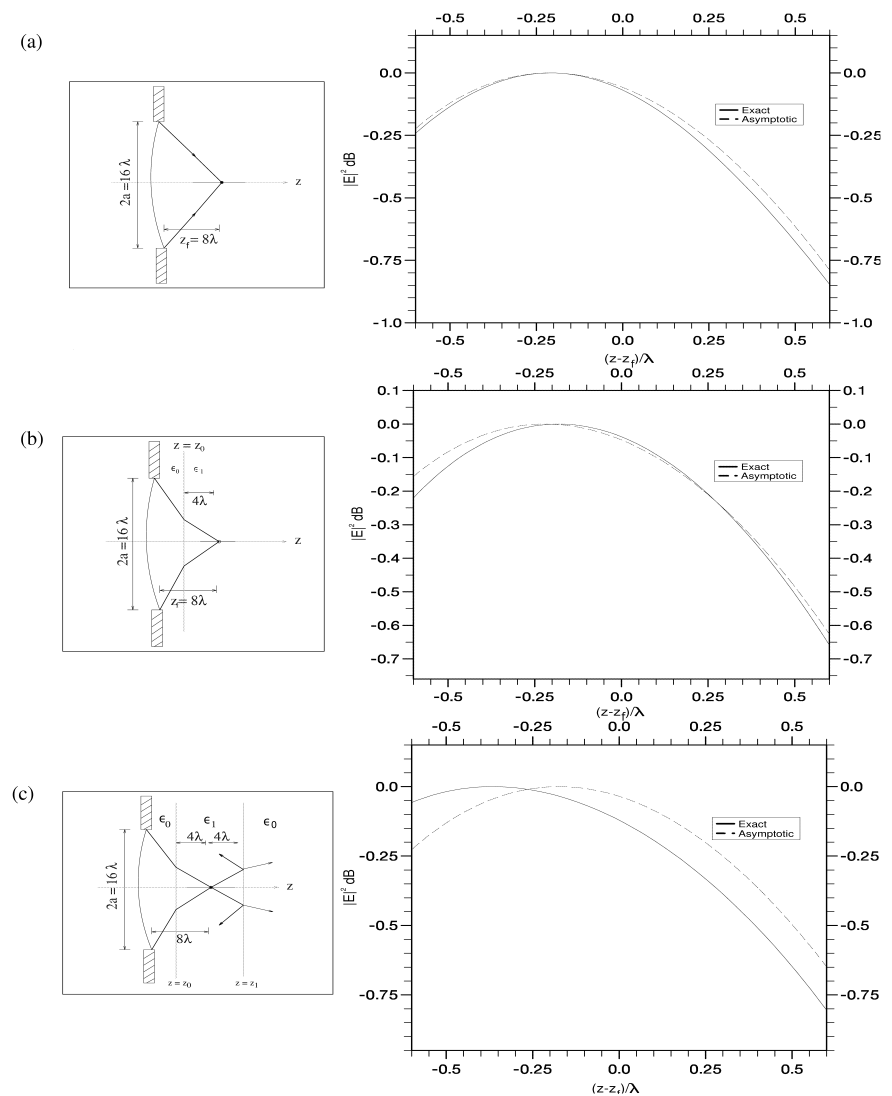

FIC. 4 Exact and asymptotic results for $|\mathbf{E}|^{2}$ along the optical axis for focusing (a) in a single medium with $\epsilon_{0}=1$, (b) through a single interface with $\epsilon_{0}=1$ and $\epsilon_{1}=1.4$, and (c) into a dielectric slab with $\epsilon_{0}=\epsilon_{2}=1$ and $\epsilon_{1}=1.4$. Here $z_{f}=8 \lambda$, $a=8 \lambda, z_{0}=4 \lambda, z_{1}=12 \lambda, N=a^{2} / \lambda z_{f}=8$, and $f$-number $=z_{f} / 2 a=0.5$, $\lambda=0.633 \times 10^{-6} \mathrm{~m}$. The incident field was polarized in the $x$ direction.

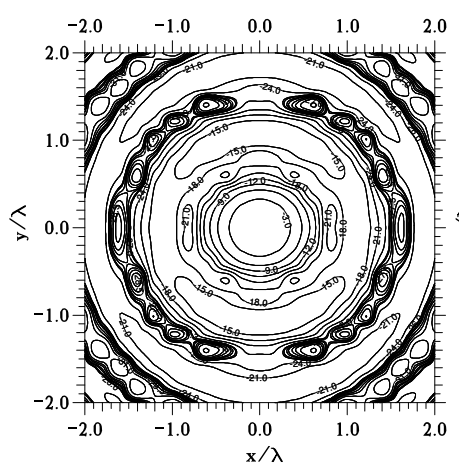

(a)

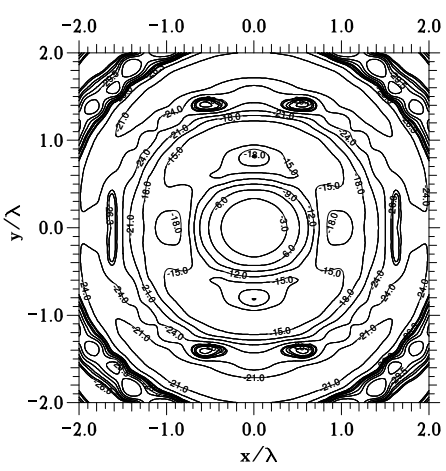

(b)
FIG. 5 Contour plots for $|\mathbf{E}|^{2}$ in the focal plane for focusing in a single medium with the same geometry as in Fig. 4, showing (left) exact results and (right) asymptotic results.

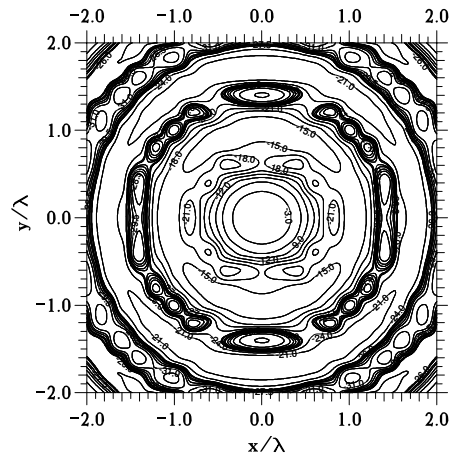

(a)

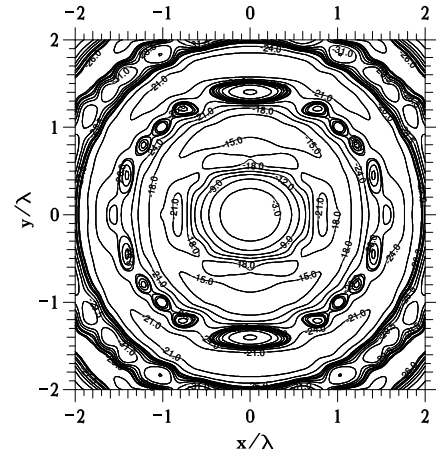

(b)
FIG. 6 Contour plots for $|\mathbf{E}|^{2}$ in the focal plane for focusing through a single interface with the same geometry as in Fig. 4, showing (a) exact results and (b) asymptotic results.

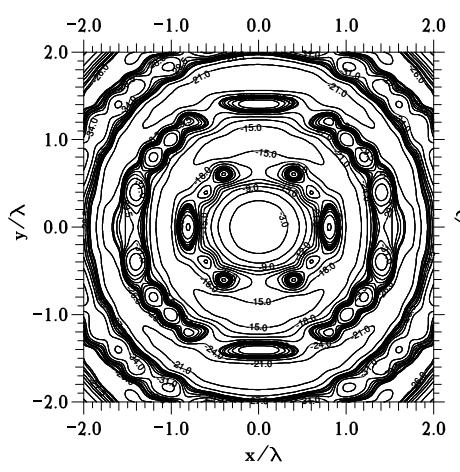

(a)

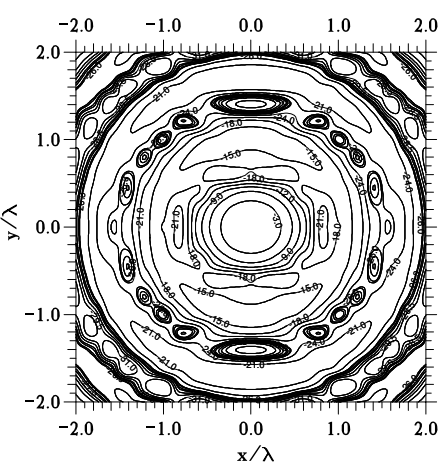

(b)
FIG. 7 Contour plots for $|\mathbf{E}|^{2}$ in the focal plane for focusing inside a dielectric slab with the same geometry as in Fig. 4, showing (a) exact results and (b) asymptotic results.

\section{Numerical Results}

In this section asymptotic and exact Kirchhoff results are presented for the focused electric field inside the slab, and for the special cases in which the slab reduces to a single interface or a single medium. All numerical results are for a focusing system of Fresnel number $N=8$ and $f$-number $=0.5$. We considered a dielectric slab with a thickness of $8 \lambda$ and an aperture radius of $a=8 \lambda$. The first interface of the slab was assumed to be at a distance of $z_{0}=4 \lambda$ from the aperture plane $z=0$. In all cases, the zeroth medium to the left of the slab and the second medium to the right of the slab were assumed to be air with $\epsilon_{0}=\epsilon_{2}=1$, and the first or slab medium was assumed to have a dielectric constant of $\epsilon_{1}=1.4$. This choice was made as a compromise to represent different slab materials, such as biological tissues with refractive indices between 1.4 and 1.45, $\mathrm{Si}$ substrates in thin films with refractive indices varying from 1.2 to 1.48 , minerals with refractive indices in the range from 1.4 to 2 , and ice with a refractive index of about 1.31. In Figs. $4-11$, the electric field is normalized to $0 \mathrm{~dB}$ at the focus, and the results pertain to a region near the focus in order to clearly see the differences between exact and asymptotic results for the focused field. All numerical results were obtained by using the phase function $\phi\left(x^{\prime}, y^{\prime}\right)$ in (12) to compensate for aberrations introduced on refraction through the first interface of the slab at $z=z_{0}$. Figures 4(a)-(c) show asymptotic and exact Kirchhoff results for the absolute value squared of the focused electric field along the optical axis, inside the slab in (c) and for the special cases in which the slab reduces to a single interface in (b) or a single medium in (a). In all three cases, both the asymptotic and exact results reveal a focal shift towards the aperture, a common feature for focusing systems of low Fresnel numbers. The difference between asymptotic and exact results is seen to be small in the case of focusing in a single medium or through a single interface, while it is significant in the case of focusing into a slab. For the case of focusing through a single interface, Fig. 4(b) shows that the difference between asymptotic and exact results is larger when the observation point lies between the interface and the focus. For this particular geometry, the asymptotic results are found to deviate by $1.3 \%$ and $0.8 \%$ from the exact results at observation points that lie $3.4 \lambda$ and $4.6 \lambda$, respectively, from the interface. Although not shown in Fig. 4(b), our numerical comparisons revealed that the difference between asymptotic and exact results increased as the observation point approached the interface, and at an observation point that was $0.5 \lambda$ from the inter- 


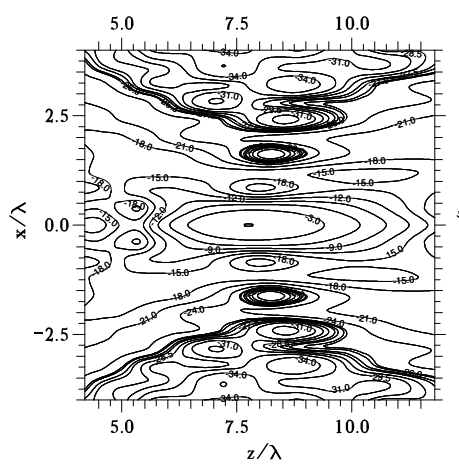

(a)

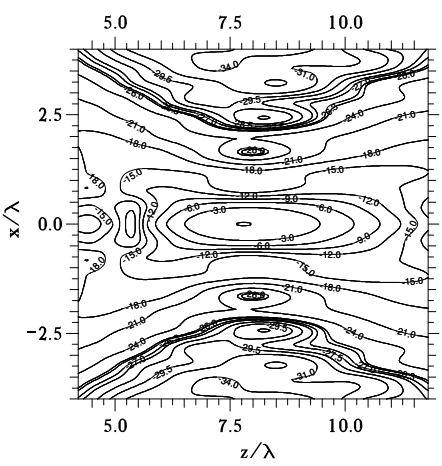

(b)
FIG. 8 Contour plots for $|\mathbf{E}|^{2}$ in the $x z$ plane for focusing in a single medium with the same geometry as in Fig. 4, showing (a) exact results and (b) asymptotic results.

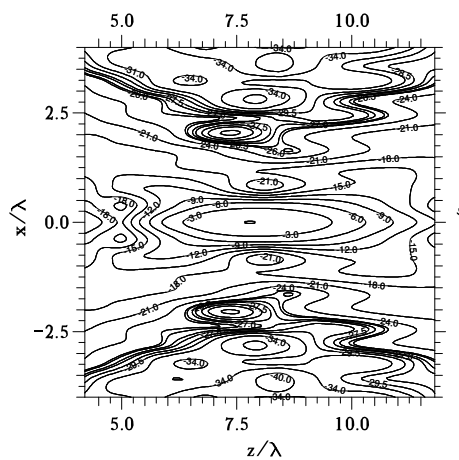

(a)

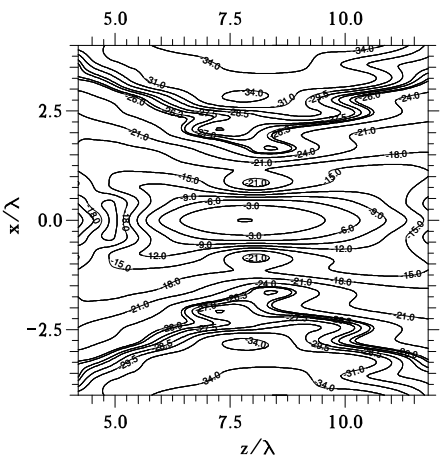

(b)
FIC. 9 Contour plots for $|\mathbf{E}|^{2}$ in the $x z$ plane for focusing through a single interface with the same geometry as in Fig. 4, showing (a) exact results and (b) asymptotic results.

face, the difference was found to be $1.8 \%$. Farther away from the interface the difference was found to gradually decrease to become less than $0.1 \%$ when the observation point was at a distance of $6 \lambda$ from the interface. At the focal point, both the exact and asymptotic results gave about the same result with a deviation less than $0.1 \%$.

Figure 4(c) shows a clear difference between exact and asymptotic Kirchhoff results for the absolute value squared of the electric field near the two interfaces of the slab. The difference is $3.8 \%$ at an observation point that lies $3.4 \lambda$ away from the first interface, and is about $3.5 \%$ at an observation point that lies $3.4 \lambda$ away from the second interface. Since the dielectric slab had a thickness of only $8 \lambda$, contributions from surface waves, which are not accounted for in the asymptotic results, may explain the difference between exact and asymptotic results. Analogously to the case of focusing through a single interface, the difference between exact and asymptotic results is seen to decrease with increasing distance between the observation point and each of the two interfaces. Figure 4 (c) shows that the asymptotic solution gives a focal shift of $0.23 \lambda$, whereas the exact solution gives a focal shift of $0.32 \lambda$.

Figures 5, 6, and 7 show (a) exact and (b) asymptotic Kirchhoff results for the absolute value squared of the focused electric field in the focal plane for the case of focusing in a single medium, through a single interface, and into a slab, respectively. The electric-field intensities are normalized to $0 \mathrm{~dB}$ at the focus, and the contours are plotted on a decible scale with isophotes being $3 \mathrm{~dB}$ apart. In all three cases, the ex-

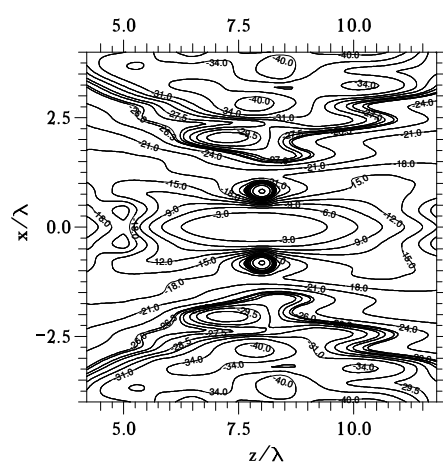

(a)

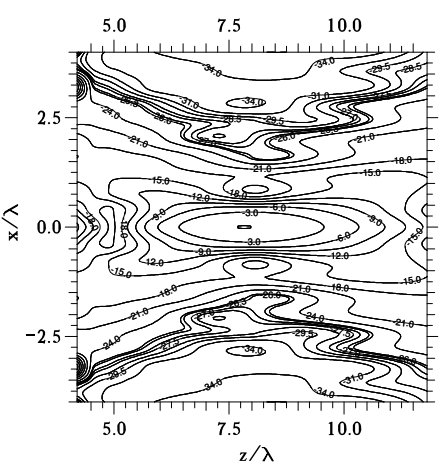

(b)
FIG. 10 Contour plots for $|\mathbf{E}|^{2}$ in the $x z$ plane for focusing inside a dielectric slab with the same geometry as in Fig. 4, showing (a) exact results and (b) asymptotic results.

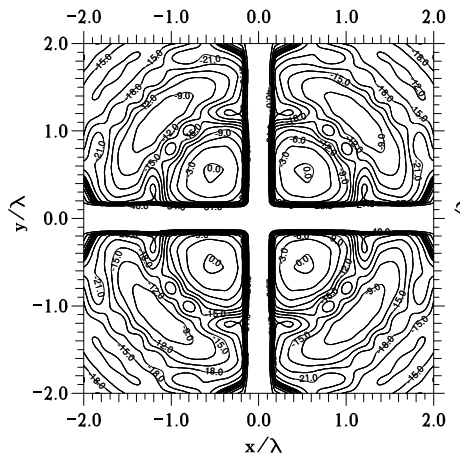

(a)

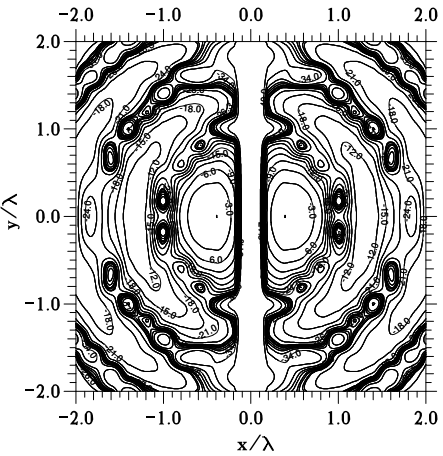

(b)
FIG. 11 Contour plots for (a) $\left|E_{y}\right|^{2}$ and (b) $\left|E_{z}\right|^{2}$ in the focal plane for focusing through a single interface with the same geometry as in Fig. 4 .

act and asymptotic results are found to give the same $3 \mathrm{~dB}$ width, but the exact results to give better concentration of the electric energy around the focus, the electric-energy contours of the asymptotic results being more elliptical. A comparison Fig. 6 for focusing in a single medium with Fig. 7 for focusing through a single interface, shows that the $3 \mathrm{~dB}$ width is smaller in the latter figure, making the energy more concentrated around the focus in the case of focusing into a dielectric medium with $\epsilon_{1}=1.4$. The exacts results for the absolute value squared of the focused field in the focal plane show that the $3 \mathrm{~dB}$ width is about $0.75 \lambda$ for focusing in a single medium (Fig. 5), whereas it is $0.64 \lambda$ for focusing through a single interface (Fig. 6) or into a slab (Fig. 7). In all three cases, the elliptical contours near the focus are elongated along the $x$ direction, i.e. along the direction of polarization of the source current in the aperture. Figures 8, 9, and 10 show contour plots for the absolute value squared of the focused electric field in the $x z$ plane for focusing in a single medium, through a single interface, and into a slab, respectively. In all three cases, both asymptotic and exact results correctly predict the asymmetric behavior of the focused field about the focal plane, which is a common feature in systems of low Fresnel numbers [14] [37][41]. Figure 8 shows that the difference between asymptotic and exact results is small for focusing in a single medium, while Figs. 9-10 show that it becomes significant for focusing through a single interface or into a slab. Both for focusing through a single interface (Fig. 9) and into a slab (Fig. 10), the exact results in (a) have contours of $|\mathbf{E}|^{2}$ that are more concentrated around the focus than those of the asymptotic results in (b). In the $x z$ plane, $|\mathbf{E}|^{2}$ is more concentrated for focusing into a slab than for focusing through a single interface or in a sin- 
gle medium. The $3 \mathrm{~dB}$ width of $|\mathbf{E}|^{2}$ in the $x z$ plane is nearly $3.00 \lambda$ for focusing in a single medium (Fig. 8), while it is $2.85 \lambda$ for focusing through a single interface (Fig. 9) and $2.6 \lambda$ for focusing into a slab (Fig. 10).

Figures 11 show (a) the $y$ or cross-polarized component and (b) the $z$ or longitudinally polarized component of the focused electric field in the focal plane for focusing from air with $\epsilon_{0}=1$ through a single interface into a dielectric medium with $\epsilon_{1}=1.4$. Figure 11(a) shows that the $y$ or cross-polarized component has a four-fold symmetry with four peaks, one in each quadrant of the focal plane, while the $z$ or longitudinallypolarized component in Fig. 11(b) is seen to have a two-fold symmetry with two peaks, one on either side of the $x$ axis, which coincides with the polarization direction of the incident field. These results are in agreement with previous findings pertaining to focusing in a single medium [1, 42]. Since the incident field was polarized in the $x$ direction, the $x$ or copolarized component of the focused electric field contained most of the energy, as expected. The $y$ and $z$ components of the focused electric field contributed only about $0.5 \%$ and $1.0 \%$, respectively, to the total focused electric energy.

\section{Conclusions}

We have examined the focusing of electromagnetic waves inside a slab and also considered the two special cases in which the slab reduces to a single interface or a single medium. To that end, we used the exact Kirchhoff solutions for the fields inside a slab given in [26] to develop efficient numerical solutions for the focused electric field. Numerical results were presented, both for exact and asymptotic Kirchhoff solutions, and the validity of the latter was discussed.

Asymptotic Kirchhoff results were obtained by expressing the impulse response, i.e. the field produced inside the slab by a point source in the aperture, as a plane-wave expansion and evaluating the associated spectral double integral by the method of stationary phase $[26,35]$. Since this asymptotic approach gives a significant reduction in computing time, we examined its range of validity. The asymptotic Kirchoff results were found to be accurate for focusing in a single medium provided that $k R \gg 1$, where $k$ is the wave number and $R$ is the distance from an aperture point to the observation point. The exact and asymptotic Kirchhoff solutions for focusing through a single interface were found to agree well, except at observation points near to the interface. This is in agreement with the findings in [35], where an accuracy better than $7.8 \%$ was obtained for an aperture-interface distance of $0.5 \lambda$ when the total distance from the aperture to the focal point exceeded $8 \lambda$, and where the accuracy was found to improve with increasing aperture-interface distance.

We considered a geometry where the aperture-interface distance was kept at $4 \lambda$, and where the total distance from the aperture to the focal point was kept at $8 \lambda$. This geometry explains the small difference found between the exact and asymptotic Kirchhoff solutions in the case of focusing through a single interface.
Our numerical comparisons showed a small difference between the asymptotic and exact Kirchhoff results for the focused electrical field inside a dielectric slab, especially at observation points near to one of the interfaces. This difference is believed to be due to contributions from surface waves, which are not accounted for in the asymptotic Kirchhoff solutions.

At low Fresnel numbers focal-shift phenomena and asymmetries were observed in each of the three cases (single medium, single interface, and slab), both in the exact and asymptotic Kirchhoff solutions. In the case of focusing through a single interface, the $y$ component and the $z$ component of the electric field in the focal plane were found to have four-fold and two-fold symmetries, respectively, in accordance with previous findings.

\section{Appendix A}

The components of the vectors $\mathbf{B}^{ \pm}(t)$ and $\mathbf{D}^{ \pm}(t)$ in (32) are given by

$$
\begin{aligned}
& B_{x}^{+}(t)=2 \frac{\omega \mu_{0}}{c^{2}} k_{z_{1}}\left[\frac{T^{T E+}}{k_{z_{0}}} \sin ^{2} \phi+\frac{k_{z_{1}}}{k_{0} k_{1}} T^{T M+} \cos ^{2} \phi\right], \\
& B_{x}^{-}(t)=2 \frac{\omega \mu_{0}}{c^{2}} k_{z_{1}}\left[\frac{r_{12}^{T E} T^{T E+}}{k_{z_{0}}} \sin ^{2} \phi\right. \\
& \left.-\frac{k_{z_{1}}}{k_{0} k_{1}} r_{12}^{T M} T^{T M+} \cos ^{2} \phi\right], \\
& D_{x}^{+}(t)=\frac{\omega \mu_{0}}{c^{2}} k_{z_{1}}\left[\frac{T^{T E+}}{k_{z_{0}}}-\frac{k_{z_{1}}}{k_{0} k_{1}} T^{T M+}\right] \cos 2 \phi, \\
& D_{x}^{-}(t)=\frac{\omega \mu_{0}}{c^{2}} k_{z_{1}}\left[\frac{r_{12}^{T E} T^{T E+}}{k_{z_{0}}}+\frac{k_{z_{1}}}{k_{0} k_{1}} r_{12}^{T M} T^{T M+}\right] \cos 2 \phi, \\
& B_{y}^{+}(t)=-D_{y}^{+}(t)=\frac{\omega \mu_{0}}{c^{2}} k_{z_{1}}\left[+\frac{k_{z_{1}}}{k_{0} k_{1}} T^{T M+}-\frac{T^{T E+}}{k_{z_{0}}}\right] \\
& \times \sin 2 \phi \text {, } \\
& B_{y}^{-}(t)=-D_{y}^{-}(t)=\frac{\omega \mu_{0}}{c^{2}} k_{z_{1}}\left[-\frac{k_{z_{1}}}{k_{0} k_{1}} r_{12}^{T M} T^{T M+}\right. \\
& \left.-\frac{r_{12}^{T E} T^{T E+}}{k_{z_{0}}}\right] \sin 2 \phi, \\
& B_{z}^{ \pm}(t)=0 ; D_{z}^{+}(t)=-i \frac{\omega \mu_{0}}{c^{2}} \frac{T^{T M+} k_{z_{1}}}{k_{0} k_{1}} \rho t^{2} \cos \phi, \\
& D_{z}^{-}(t)=-i \frac{\omega \mu_{0}}{c^{2}} \frac{r_{12}^{T M} T^{T M+} k_{z_{1}}}{k_{0} k_{1}} \rho t^{2} \cos \phi .
\end{aligned}
$$

\section{Appendix B}

Consider first the two integrals $\mathbf{F}_{H j}^{ \pm}$in (35), where the integration variable $t$ is in the interval $[0,1]$, and let $t=\sin u$ to give

$$
\mathbf{F}_{H j}^{ \pm}=k_{1} \int_{u_{j}^{-}}^{u_{j}^{+}} \mathbf{A}_{H j}^{ \pm}(u) \exp \left[i g_{H j}^{ \pm}(u)\right] d u, \quad ; \quad(j=1,2),
$$


where

$$
\begin{gathered}
u_{1}^{-}=0 ; u_{1}^{+}=u_{2}^{-}=u_{r}=\sin ^{-1}\left(k_{r}\right), ; u_{2}^{+}=\pi / 2, \\
g_{H j}^{ \pm}(u)=k_{1}\left[z_{0} \sqrt{k_{r}^{2}-\sin ^{2} u}\right]+g_{H 2}^{ \pm}(u), \\
g_{H 2}^{+}(u)=k_{1}\left(z-z_{0}\right) \cos u ; g_{H 2}^{-}(u)=k_{1}\left(d+z_{1}-z_{0}\right), \\
\mathbf{A}_{H 1}^{ \pm}(u)=\left[\mathbf{B}^{ \pm}(t) J_{0}\left(\beta_{1} \sin u\right)\right. \\
\left.\quad+\mathbf{D}^{ \pm}(t) \frac{2 J_{1}\left(\beta_{1} \sin u\right)}{\beta_{1} \sin u}\right] \sin u, \\
\mathbf{A}_{H 2}^{ \pm}(u)=\mathbf{A}_{H 1}^{ \pm}(u) \exp \left(-k_{1}\left[z_{0} \sqrt{k_{r}^{2}-\sin ^{2} u}\right]\right) .
\end{gathered}
$$

Here $\mathbf{B}^{ \pm}(t)$ and $\mathbf{D}^{ \pm}(t)$ in (48) follow from (36)-(43) with the substitutions

$$
t \rightarrow \sin u ; k_{z_{0}} \rightarrow k_{1} \sqrt{k_{r}^{2}-\sin ^{2} u} ; k_{z_{1}} \rightarrow k_{1} \cos u,
$$

while $\mathbf{B}^{ \pm}(t)$ and $\mathbf{D}^{ \pm}(t)$ in (49) follow from (36)-(43) with the same substitutions as in (50) except that

$$
k_{z_{0}} \rightarrow i k_{1} \sqrt{\sin ^{2} u-k_{r}^{2}}
$$

Consider next the integral $\mathbf{F}_{I}^{ \pm}$in (35), where $t>1$, and let $t=\cosh u$ to obtain

$$
\mathbf{F}_{I}^{ \pm}=-i k_{1} \int_{0}^{u_{N}^{ \pm}} \mathbf{A}_{I}^{ \pm}(u) \exp \left(-g_{I}^{ \pm}(u)\right) d u,
$$

where

$$
\begin{gathered}
g_{I}^{+}(u)=k_{1}\left[z_{0} \sqrt{\cosh ^{2} u-k_{r}^{2}}+\left(z-z_{0}\right) \sinh u\right], \\
g_{I}^{-}(u)=k_{1}\left[z_{0} \sqrt{\cosh ^{2} u-k_{r}^{2}}+\left(d+z_{1}-z\right) \sinh u\right], \\
\mathbf{A}_{I}^{ \pm}(u)=\left[\mathbf{B}^{ \pm}(t) J_{0}\left(\beta_{1} \cosh u\right)\right. \\
\left.\quad+\mathbf{D}^{ \pm}(t) \frac{2 J_{1}\left(\beta_{1} \cosh u\right)}{\beta_{1} \cosh u}\right] \cosh u
\end{gathered}
$$

with $\mathbf{B}^{ \pm}(u)$ and $\mathbf{D}^{ \pm}(u)$ following from (36)-(43) with the substitutions

$$
t \rightarrow \cosh u ; k_{z_{0}} \rightarrow i k_{1} \sqrt{\cosh ^{2} u-k_{r}^{2}} ; k_{z_{1}} \rightarrow i k_{1} \sinh u .
$$

The upper integration limit $u_{N}^{ \pm}$in (52) is determined by the requirement that

$$
\exp \left\{-k_{1} z_{0}\left[\sqrt{\cosh ^{2} u_{N}^{ \pm}-k_{r}^{2}}+C^{ \pm} \sinh u_{N}^{ \pm}\right]\right\}=10^{-N}
$$

where $C^{+}=\left(z-z_{0}\right) / z_{0}$ and $C^{-}=\left(d+z_{1}-z\right) / z_{0}$. This gives

$$
u_{N}^{ \pm}=\sinh ^{-1}\left(\Sigma_{N}^{ \pm}\right)=\ln \left(\Sigma_{N}^{ \pm}+\sqrt{\left(\Sigma_{N}^{ \pm}\right)^{2}+1}\right)
$$

where

$$
\Sigma_{N}^{ \pm}=\left\{\begin{array}{ll}
\frac{A_{N}^{ \pm}-\epsilon_{N} C^{ \pm}}{1-\left(C^{ \pm}\right)^{2}} & \text { for } C^{ \pm}<1, \epsilon_{N}^{2}>1-k_{r}^{2} \\
\frac{A_{N}^{ \pm}+\epsilon_{N} C^{ \pm}}{\left(C^{ \pm}\right)^{2}-1} & \text { for } C^{ \pm}>1, \epsilon_{N}^{2}<1-k_{r}^{2}
\end{array},\right.
$$

with

$$
\begin{aligned}
A_{N}^{ \pm} & =\sqrt{\left(\epsilon_{N} C^{ \pm}\right)^{2}+\left|\left[1-\left(C^{ \pm}\right)^{2}\right]\left(\epsilon_{N}^{2}+k_{r}^{2}-1\right)\right|} \\
\epsilon_{N} & =\frac{N \ln 10}{k_{1} z_{0}} .
\end{aligned}
$$

When $C^{ \pm}=1$, we have $\epsilon_{N}^{2}>1-k_{r}^{2}$ and

$$
\begin{aligned}
u_{N}^{+}=u_{N}^{-}= & \sinh ^{-1}\left[\frac{\epsilon_{N}^{2}+k_{r}^{2}-1}{\epsilon_{N}}\right] \\
= & \ln \left[\epsilon_{N}^{2}+k_{r}^{2}-1+\sqrt{\left(\epsilon_{N}^{2}+k_{r}^{2}-1\right)^{2}+\epsilon_{N}^{2}}\right] \\
& -\ln \epsilon_{N} .
\end{aligned}
$$

\section{Appendix C - Integration Algorithms}

In order to reduce the computing time for numerical evaluation of the integrals in (44) and (52), we use the GaussLegendre method [36] to compute each integral for which the integrand contains no rapidly oscillating exponential function and the SSP (Stamnes-Spelkavik-Pedersen) method [32,34] to compute each integral for which the integrand is a product of an amplitude function and an oscillating exponential function that varies fast compared to the amplitude function.

As an example, consider the integral $\mathbf{F}_{H 1}^{ \pm}$in (44). When the argument $\beta_{1} \sin u$ of the cylindrical Bessel functions $J_{0}$ and $J_{1}$ in (48) is small, i.e. when $\beta_{1} \sin u \leq 10$, the amplitude function $\mathbf{A}_{H 1}^{ \pm}(u)$ in (48) oscillates so slowly compared with the exponential function $\exp \left[i g_{H 1}^{ \pm}(u)\right]$ in (44) that we may use the SSP method to compute the integrals in (44). When $\beta_{1} \sin u>10$, we replace the Bessel functions in (48) with their asymptotic series, and divide the integral in (44) with $j=1$ in three parts, i.e.

$$
\mathbf{F}_{H 1}^{ \pm}=\mathbf{F}_{H 1,1}^{ \pm}+\mathbf{F}_{H 1,+}^{ \pm}+\mathbf{F}_{H 1,-}^{ \pm},
$$

where

$$
\mathbf{F}_{H 1,1}^{ \pm}=\int_{0}^{u^{\prime}} \mathbf{A}_{H 1}^{ \pm}(u) e^{i g_{H 1}^{ \pm}(u)} d u ; u^{\prime}=\sin ^{-1}\left(5 / \beta_{1}\right) .
$$

Here $\mathbf{A}_{H 1}^{ \pm}(u)$ and $g_{H 1}^{ \pm}(u)$ are given by (48) and (46) with $j=1$, and $\mathbf{F}_{H 1, q}^{ \pm}$(with $q=+,-$ ) is given by

$$
\mathbf{F}_{H 1, q}^{ \pm}=\int_{u^{\prime}}^{u_{r}} \mathbf{A}_{H 1, q}^{ \pm}(u) e^{i g_{H 1, q}^{ \pm}(u)} d u ; u_{r}=\sin ^{-1}\left(k_{r}\right),
$$

where

$$
g_{H 1, q}^{ \pm}(u)=g_{H 1, q}^{ \pm}(u)+q \beta_{1} \sin u
$$

$$
\mathbf{A}_{H 1, q}^{ \pm}(u)=\mathbf{B}_{H 1}^{ \pm}(u) G_{0}^{q}\left[\beta_{1} \sin u\right]+\mathbf{D}_{H 1}^{ \pm}(u) \frac{2 G_{1}^{q}\left[\beta_{1} \sin u\right]}{\beta_{1} \sin u} .
$$


The functions $\mathbf{B}_{H 1}^{ \pm}$and $\mathbf{D}_{H 1}^{ \pm}$are given in (36)-(43) and

$$
G_{n}^{+}(x)=G_{n}(x) ; G_{n}^{-}(x)=G_{n}^{*}(x),
$$

where

$$
G_{n}(x)=\frac{\exp [-i(\pi / 4+n \pi / 2)]}{\sqrt{2 \pi x}}\left[P_{n}(x)+i Q_{n}(x)\right] .
$$

The asymptotic series $P_{n}(x)$ and $Q_{n}(x)$ are given in [36]. The SSP method is used to compute the integrals in (64).

\section{References}

[1] M. Mansuripur, "Distribution of light at and near the focus of high-numerical-aperture objectives", J. Opt. Soc. Am. A 3, 20862093 (1995).

[2] D. G. Flagello, T. Milster, and A. E. Rosenbluth, "Theory of high-NA imaging in homogeneous thin films", J. Opt. Soc. Am. A 13, 53-64 (1996).

[3] H. Ling, and S. W. Lee, "Focusing of electromagnetic waves through a dielectric interface", J. Opt. Soc. Am. A 1, 965-973 (1984).

[4] H. Ling, S. W. Lee, and W. Gee, "Frequency optimization of focused microwave hyperthermia applications", Proc. IEEE 72, 224225 (1984).

[5] P. Török, P. Varga, Z. Laczic, and G. R. Booker, "Electromagnetic diffraction of light focused through a planar interface between materials of mismatched refractive indices: an integral representation", J. Opt. Soc. Am. A 12, 325-332 (1995).

[6] P. Török, P. Varga, and G. R. Booker, "Electromagnetic diffraction of light focused through a planar interface between materials of mismatched refractive indices. I. Structure of the electromagnetic field", J. Opt. Soc. Am. A 12, 2136-2144 (1995).

[7] P. Török, P. Varga, and G. Nemeth, "Analytical solution of the diffraction integrals and interpretation of wave-front distortion when light is focused through a planar interface between materials of mismatched refractive indices", J. Opt. Soc. Am. A 12, 2660-2671 (1995).

[8] P. Török, "Focusing of electromagnetic waves through a dielectric interface by leses of finite fresnel number", J. Opt. Soc. Am. A 3, 2086-2093 (1998).

[9] J. H. Erkkila, and M. E. Rogers, "Diffracted fields in the focal volume of a converging wave", J. Opt. Soc. Am. 71, 904-905 (1981).

[10] J. J. Stamnes, and B. Spjelkavik, "Focusing at small angular apertures in the Debye and Kirchhoff approximations", Opt. Commun. 40, 81-85 (1981).

[11] E. Wolf, and Y. Li, "Conditions for the validity of the Debye integral representation of focused fields", Opt. Commun. 39, 205-210 (1981).

[12] Y. Li, "Encircled energy for systems of different Fresnel numbers", Optik 64, 207-218 (1983).

[13] Y. Li, and H. Platzer, “An experimental investigation of diffraction patterns in low-Fresnel-number focusing systems", Opt. Acta 30, 1621-1643 (1983).

[14] Y. Li, "Dependence of the focal shift on Fresnel number and $f$-number", J. Opt. Soc. Am. A 72, 770-4 (1982).

[15] V. Dhayalan, and J. J. Stamnes, "Focusing of electric-dipole waves in the Debye and Kirchhoff approximations", Pure Appl. 0pt. 6, 347-372 (1997).
[16] G. W. Farnell, "Measured phase distribution in the image space of a microwave lens", Can. J. Phys. 36, 935-943 (1958).

[17] C. A. Taylor, and B. J. Thompson, "Attempt to investigate experimentally the intensity distribution near the focus in the error-free diffraction patterns of circular and annular apertures", J. 0pt. Soc. Am. 48, 844-50 (1958).

[18] J. J. Stamnes, and V. Dhayalan, "Focusing of electric-dipole waves", Pure Appl. Opt. 5, 195-226 (1996).

[19] Q. W. Zhan, "Trapping metallic Rayleigh particles with radial polarization", 0pt. Express 12, 3377-3382 (2004).

[20] L. E. Helseth, "Focusing of atoms with strongly confined light potentials", Opt. Commun. 212, 343-352 (2002).

[21] M. Meier, V. Romano, and T. Feurer, "Material processing with pulsed radially and azimuthally polrized laser radiation", Appl. Phys. A-Matter 86, 21965-21972, (2010).

[22] R. Dorn, S. Quabis, and G. Leuchs, "Sharper focus for a radially polarized light beam", Phys. Rev. Lett. 91, 233901-233904 (2003).

[23] S. Quabis, R. Dorn, M. Eberler, 0. Glöckl, and G. Leuchs, “Focusing light to tighter spot", Opt. Commun. 179, 1-7 (2000).

[24] S. Quabis, R. Dorn, M. Eberler, 0. Glöckl, and G. Leuchs, "The focus of light - theoretical calculation and experimental tomographic reconstruction", Appl. Phys. B-Lasers 072, 109-113 (2001).

[25] H. P. Urbach, and S. F. Pereira, "The field in focus with maximum electric field components", Phys. Rev. A 79, 013825 (2009).

[26] V. Dhayalan, and J. J. Stamnes, "Focusing of electromagnetic waves into a dielectric slab I. Exact and asymptotic results", Pure Appl. 0pt. 7, 33-52 (1998).

[27] J. J. Stamnes, and H. A. Eide, "Exact and approximate solutions for focusing of two-dimensional waves. I. Theory", J. Opt. Soc. Am. A 15, 1285-1291 (1998).

[28] H. A. Eide, and J. J. Stamnes, "Exact and approximate solutions for focusing of two-dimensional waves. II. Numerical comparisons between exact, Debye, and Kirchhoff theories", J. opt. Soc. Am. A 15, 1308-1319 (1998).

[29] H. A. Eide, and J. J. Stamnes, "Exact and approximate solutions for focusing of two-dimensional waves. III. Numerical comparisons between exact and Rayleigh-Sommerfeld theories", J. Opt. Soc. Am. A 15, 1292-1307 (1998).

[30] D. Jiang and J. J. Stamnes, "Theoretical and experimental results for two-dimensional electromagnetic waves focused through an interface", Pure Appl. Opt. 7, 627-641 (1998).

[31] J. J. Stamnes, and D. Jiang, "Focusing of two-dimensional electromagnetic waves through a plane interface", Pure Appl. 0pt, 7, 603-625 (1998).

[32] J. J. Stamnes, Waves in Focal Regions, (Adam Hilger, Bristol and Boston, 1986).

[33] W. H. Press, B. P. Flannery, S. A. Teukolsky, and W. T. Vetterling, Numerical Recipes, (Cambridge Univ. Press, 1989.

[34] J. J. Stamnes, B. Spjelkavik, and H. M. Pedersen, "Evaluation of diffraction integrals using local phase and amplitude approximations", Opt. Acta 30, 207-222 (1983).

[35] V. Dhayalan, and J. J. Stamnes, "Comparison of exact asymptotic results for the focusing of electromagnetic waves through a plane interface", Appl. Opt. 39, 6332-6340 (2000).

[36] M. Abramowitz, and I. A. Stegun, Handbook of Mathematical Functions, (Dover, New York, 5th edition (1968)).

[37] Y. Li, and E. Wolf, “Three-dimensional intensity distribution near 
the focus in systems of different Fresnel numbers", J. Opt. Soc. Am. A 1, 801-808 (1984).

[38] Y. Li, "Focal shifts in small-fresnel-number focusing systems of different relative aperture", J. Opt. Soc. Am. A 20, 234-239 (2003).

[39] Y. Li, "Focal shifts in diffracted converging electromagnetic waves. I. Kirchhoff theory", J. Opt. Soc. Am. A 22, 68-76 (2005).

[40] Y. Li, "Focal shifts in diffracted converging electromagnetic waves. II. Rayleigh theory", J. Opt. Soc. Am. A 22, 77-83 (2005).

[41] J. J. Stamnes, and V. Dhayalan, "Focal shifts on focusing through a plane interface", 0pt. Commun. 282, 2286-2291 (2009).

[42] M. Mansuripur, "Certain computational aspects of vector diffraction problems", J. Opt. Soc. Am. A 6, 786-805 (1989). 